\title{
THE HYDRODYNAMICAL EVOLUTION OF GAS IN YOUNG EL- LIPTICAL GALAXIES
}

\author{
R. Kunze, H.H. Loose \& H.W. Yorke \\ University Observatory Göttingen \\ Geismarlandstraße 11 \\ D-3400 Göttingen \\ Federal Republic Germany
}

\begin{abstract}
We calculate the partial inflow of gas fuelled by stellar mass loss at an early epoch $\left(10^{9} \mathrm{yr}\right.$ after the birth of the galaxy) during the evolution of an elliptical galaxy assuming a modified King model stellar distribution. The influence of the partial thermalization of stellar mass lost on the amount of gas which can be stored in the nucleus of a typical elliptical during the time of partial inflow is investigated. Masses up to $10^{5} M_{\odot}$ of cool $\left(\leq 10^{4} K\right)$ material can be stored in the nucleus of the galaxy before the fast dissipation of the "kinetic bulk energy" of the nuclear gas cloud leads to "thermal" instability and subsequent collapse. A supermassive star can form. A detailed discussion of the model and the results is subject of a forthcoming paper (Kunze et al., 1986).
\end{abstract}

\section{INTRODUCTION}

A number of elliptical galaxies show evidence of compact central objects which power their nonthermal activity (Dressel et al., 1982). These objects are believed to have masses ranging from $10^{6}$ to $10^{8} M_{\odot}$ (massive black holes, supermassive stars, etc.). They have formed at early times in the evolution of the galaxy because active galactic nuclei are observable at high redshifts.

During early epochs of their evolution elliptical galaxies have partial inflow of gas which has its origin in the process of stellar mass loss (Mathews \& Loewenstein, 1986 and references therein). The amount of gas which can accumulate before it becomes unstable depends critically on the physics of heat input into the interstellar medium. In earlier works the energy supplied by stellar ejecta and supernova explosions is assumed to be immediately converted into internal energy of the interstellar gas (e.g. Mac Donald \& Bailey, 1981; hereafter MB). But the small amounts of ionized gas $\left(\leq 10^{6} M_{\odot}\right)$ observed in elliptical galaxies (Phillips et al., 1985) may be a clue to incomplete thermalization of stellar ejecta (White \& Chevalier, 1983). We want to check the importance of the assumption of immediate thermalization of stellar ejecta for the resultant amount of gas which can be stored in the core of the galaxy during the time when inflow occurs. 


\section{THE MODEL}

We consider a spherical elliptical galaxy (without rotational motions) where the stellar density distribution is taken from King (1972). The mass loss rate from the stars (except from supernovae) is calculated similar to MB. The mass loss rate from supernova explosions (type I) is taken from observations (Tamman, 1977).

We simulate the incomplete thermalization of stellar ejecta by the introduction of a "kinetic bulk energy" to account for the random motion of clumpy mass lost. The efficiencies $\gamma_{s n}$ and $\gamma_{\star}$ for creating kinetic bulk energy from SN events and stellar ejecta, respectively, are taken to $\gamma_{s n}=0.28$ (Spitzer, 1978) and $\gamma_{\star}=0.1$ (only $10 \%$ of the total kinetic energy of stellar ejecta is used to create random cloud motions, the remainding energy is thermalized instantaneously). We assume that the kinetic bulk energy generated by the processes outlined above is dissipated exponentially and the $e^{-}$-folding time is identified with the local dynamical time scale.

\section{RESULTS}

The evolution of the partial inflow is followed by an implicit hydrodynamical code including the generation of kinetic bulk energy with the efficiencies specified above. The calculations started from an initially gas-free state. After the gas in the nucleus of the galaxy cooled down to $\leq 10^{4} \mathrm{~K}$ the nuclear gas cloud is stabilized by the kinetic bulk energy and a shock front at $\sim 10 p c$ from the center develops. About $10^{5} M_{\odot}$ gas are gradually accumulated interior the shock. When $M_{\text {gas }}\left(r_{\text {shock }}\right) \geq$ $M_{\star}\left(r_{\text {shock }}\right)$ collapse sets in due to rapid dissipation of the kinetic bulk energy. The time-scale for the collapse is less than that for accumulation of the gas, therefore the mass of the collapsing gas remains nearly constant. A hydrostatic core forms which is bounded by an accretion shock front.

The further evolution was not followed numerically. Assuming that fragmentation does not occur while the core accretes matter from the surroundings and that all matter will be accreted which is able to fall onto the central object in $t_{f f} \sim 10^{4} y r$ we obtain a mass of $M \sim 10^{5} M_{\odot}$ for the central object.

\section{REFERENCES}

Dressel, L.L., Bania, T.M. and O'Connell, R.W.: 1982, Astrophys. J. 259, 55

King, I.R.: 1972, Astrophys. J. 174, L123

Kunze, R., Loose, H.-H. and Yorke, H.W., 1986: submitted to Astron. \& Astrophys.

MacDonald, J. and Bailey, M.E.: 1981, Monthly Notices Roy. Astron. Soc. 197, 995 (MB)

Mathews, W.G. and Loewenstein, M.: 1986, Astrophys. J. 306, L7

Phillips, M.M., Jenkins, C.R., Dopita, M.A., Sadler, E.M. and Binette, L.: 1986, Astrophys. J. 91, 1062

Spitzer, L. Jr.: 1978, in 'Physical Processes in the Interstellar Medium', John Wiley \& Sons, New York, p. $255 \mathrm{ff}$.

Tamman, G.A.: 1977, Astrophys. Space Sci. 66, 95

White, R.E. III and Chevalier, R.A.: 1983, Astrophys. J. 275, 69 